\title{
A Review on Face masks - Current Scenario and Future Aspect
}

\author{
Syeda Haseen Buvabi* and Vani Srinivasan \\ Department of Chemistry, Mount Carmel College, Bengaluru, Karnataka, India. \\ *haseenbuvabi@yahoo.com²vanis2410@gmail.com
}

\begin{abstract}
Viruses and bacteria have posed a significant threat in various parts of the planet at various times. However, in 2020, the entire world was affected at the same time in such a way that it changed the very foundation of how we live, work, and interact with one another. Never before in history has the entire human race been so vulnerable at the same moment. The most economical preventive measures for most people all over the world during this period were sanitizers and face masks. Many different types of face masks have been fabricated keeping various factors like their antimicrobial effectiveness, reusability, eco-friendly method of manufacturing, cost-effectiveness and most importantly biodegradability. This paper gives an overview of those materials which are used in face masks which could be synthesized easily and affordably. The future perspective of the masks is also briefly discussed keeping the smart and sensor based devices on the rise, providing information for researchers looking for ways to synthesise face masks that would satisfy the needs of the circumstance.
\end{abstract}

Key Words: Face masks, biodegradable, antimicrobial, reusable

\section{Introduction}

The basic requirement of a face mask during the pandemic is its effectiveness against microbes. But it doesn't stop there. A normal face mask should satisfy many parameters like it should be comfortable for the wearer i. e the breathability, affordability, reusability and of course, its biodegradability (1). For this sake the masks must be put to various tests like, resistance to air flow, differential pressure test, fluid resistance test, particulate filtration test, bacterial filtration test and antimicrobial tests.

Majorly face masks are classified as surgical and respiratory. Respiratory masks are specially suitable for highrisk environment and is very effective against viruses and bacteria, whereas, surgical masks do not protect much. Interception, diffusion and electrostatic attraction and inertial impaction could be the most probable mechanisms of working of face masks (2). A surgical mask is usually comprised of layers: inner layer of soft nonwoven absorbent, central layer of a melt-blown fibre, and an outer layer of unwoven hydrophobic material. Each layer has a specific function: the inner layer is purposed to absorb moisture, sweat, and the spit of the wearer; the middle layer of the surgical mask is designed as an electret filter to prevent germs from coming in or exiting from the mask; and the outer layer is purposed to repulse water, sweat etc. The N95 respirator is comprised of many layers of PP nonwoven fabric. The two external protective layers are produced using a spun bound to cover both the inner and outer of the N95 respirator. There is a layer of pre-filtration between these spun bound layers. The last layer is a nonwoven melt-blown electret material of high-quality that controls filtration competence (3) There are two ways of making the face masks, Melt Blowing and electro spinning method. The melt blown process is a single-step method for converting polymeric raw materials into unwovens fibers. This method is popular as it is easy and flexible (4). Electro spinning is a method that helps in the synthesis of unwoven nanofibers. The method is useful for all kinds of polymers (alloys), metals and ceramics. Many biodegradable polymers have been synthesized but this method to yield nanofibers to prepare reusable face masks (5). To achieve this many metals and metal oxide nano particles which have been time-tested for their antimicrobial action have been used extensively either as such or as nano composites for making face 
masks. Silver, Copper and zinc being the most favourable metals for this purpose. Other metal oxides like Aluminium Oxide, $\mathrm{TiO} 2$ and $\mathrm{MgO}$ also have been found to be very active when supported together as composites. (6). As there are various other concerns like their biodegradability which already has become a major environmental concern all over the world (7), biodegradable polymer-based face masks are synthesized (8). In fact, biodegradable polymers blended with metals/metal oxide nanoparticles-based face masks are the most widely studied in the current research articles. Such polymer blended face masks have been reported to have all the requirements of a good face mask. To give reusability and hydrophobicity, many graphene based face masks have been synthesized. Recent articles focus more on nano-sensor based smart-face masks, natural product origin based and photosensitive face masks with auto cleaning ability all of which are discussed briefly in this review.

\section{Metals and Metal oxide-nanoparticles based facemask}

The antibacterial activity of $\mathrm{Ag}$ supported by $\mathrm{SiO} 2, \mathrm{ZnO}$, and $\mathrm{CuO}$ on a glass surface was investigated by Padryk Merkl et al (9). Nano silver, out of the three nano materials tested, had the best antiviral efficacy against SARS-CoV-2, lowering viral load by up to $75 \%$ after 5 minutes and 98 percent after two hours, indicating nano silver's potential as an antiviral coating. The layer of $\mathrm{ZnO}$ nanoparticles does this. The virus stability does not appear to be affected by the $\mathrm{ZnO}$ nanoparticle layer over time. The $\mathrm{CuO}$ nanoparticle film, on the other hand, appears to have a considerable impact. After 30 minutes, the viral load has decreased by 54\%, and after 120 minutes, it has reached its lowest level. A reduction of up to $76 \%$ is possible. Melissa and Blevens et al (10) evaluated the antiviral and antimicrobial potency of two select brands of face masks and found them to be relatively authentic in their claim. Of the two masks one used AgION technology and the other one was three layered antiviral cloth and both were patented materials. Abhishek Kumar et al (11) synthesized Copper Nano Wire coated with Zeolitic Imidazole Framework, Cu@ZIF-8 NW functionalized filters, using a copolymer for the stabilization. This copolymer is also found to render the surface passive. The authors studied the antiinflammatory, antibacterial and antiviral properties and found it to be a promising material for face masks as they had the advantage of sustained release of zinc ion along with copper which gives a self-sanitizing effect to the material which was missing in either Copper Nano Wire face mask or ZIF face mask separately. Also this material was found to be thermally more stable and more reusable compared to the masks made with either $\mathrm{CNW}$ or with ZIF.

Sunghoon Jung et al(12) studied the Copper thin film based face mask.Copper deposition was performed using a direct current (DC) magnetron sputtering system with high-purity copper as the sputtering target. But since film adhesion on poly propylene material improved on oxygen ion beam pretreatment, the copper gets converted to cuprous oxide. This study also compares the ability of copper release from the surface with their prevouos work and report that this method of making thin film reduced the risk of detached copper nanoparticles to be absorbed in the respiratory tract. Motahira Hashmi et al (13)synthesized copper oxide loaded PAN material and studied their properties extensively and recommended this as an efficient antibacterial and breathable material for face masks.

Chaitanya B. Hiragond et al(14) synthesized colloidal AgNP via simple reduction of Ag solution of 10,000ppm with hydrazine hydrate. The face masks were coated with these colloidal AgNP by simple dipping method and their antibacterial studies were done. These face masks showed good antibacterial property suggesting this is a very simplest method of making AgNP based face masks. They are cost effective as only 100ppm colloidal solution was enough to make the mask effective against bacteria. Affaf Khamis Aloufy and Magdi AbdelMoneim El-Messiry (15) synthesized AgNP loaded nylon 6 material for facemask and analysed its anti microbial property and found it to be very effective. Benjamin Valdez-Salas et al (16) prepared AgNp based face masks by synthesizing AgNP by electrochemical method. This material was tested against H5N1 virus and was found to be $99.9 \%$ effective . It also showed very good antimicrobial activity against wide range of bacteria. This method involves affordable method of synthesis of AgNP. Gownolla Malegowd Raghavendra et al (17) studied step-reduced synthesis of AgNP from starch and distilled water by microwave irradiation. The initial step of solubilizing starch has been avoided. They were successful in synthesizing 5-10nm spherical AgNP with good microbial activity. This method offers a simplified approach to make face masks. Aniruddha Adhikari et al (18) synthesized Zinc Oxide Nano Flowers(ZnONF) loaded cotton fabric and did an eleborate spectroscopic, 
computational and antibacterial studies. Their findings showed that this material could be used as a good reusable face mask or even as Protective material.

\section{Biodegradable polymer based Face masks with nano composites}

Vigneshwaran Shanmugam et al (19) et al very recently have elaborately discussed the various biodegrable materials from natural products like cellulose,chitosan, alginate, gelatin, silk fibroin, collagen, keratin and prolamine based proteins. They have suggested the doping of these materials with silver nanoparticles t improve their antimicrobial property. Vahid Babaahmadi et al (20) have shown the development of biodegradable polymers especially synthetic ones and natural polymers for making face masks. Chitosan, alginate, collagen, and gelatin are the source for natural and polyvinyl alcohol, polyethylene oxide, polycaprolactone, and polylactic acid are the ones preferred from synthetic polymers. The usual methods of making face masks are meltspinning, wet-spinning and/or electrospinning. Natural fibres were found to have good biocompatibility while better mechanical properties are shown by synthetic fibres. Various multifunctional properties like, antimicrobial, air/water permeability, comfort and wearability, self-cleaning ability, reusability and biodegradability have been discussed with tabulation. Pintu Pandit et al (21) have discussed about the various properties and applications of different categories of cellulose based face masks. Various antiviral-coated face masks of different materials, where some of them plant origin based products, are applied on natural textile materials to enhance the functionality of face masks against virus and other fungus. Gluten can be electrospun as such or blended with poly vinyl alcohol, therefore, gluten blended PVA membrane showing maximium filtration efficiency has also been reported. It has been observed that gluten can be chemically modified and used as ultrafine nanoweb filter media (Capezza et al., 2020).

Sejin Choi et al (22) synthesized a Janus membrane filter impregnated with poly(butylene succinate)-based (PBS-based) micro and nanofiber mats synthesized by electrospinning method. The nanoscale and microscale fibers are useful in capturing the PM and reducing the pressure drop respectively. It is completely biodegraded in four weeks. Morganti P and Morganti G (23)and group obtained the natural tissue based fibre containing very less percentage of silver in nano dimension, which was found to be very effective against bacteria.

\section{Cellulose nanocomposite-based face masks}

Cellulose acetate and a polyvinylidene fluoride nanofiber layer were developed by C.Akdumen (24) with complete filtration that can be used in N95 respirators. Alyne R. de Araújo et al (25) presented the comparison of different filtering materials made with cellulose. All the parameters required for an efficient face mask have been discussed in the paper. According to the study cotton-based filter material is found to be best for the reusable mask as it is biodegradable and low cost. Yogesh Khairnar et al (26) have discussed in detail about the cellulose-based bio nanocomposites. They have studied them under two catogories namely Cellulose with synthetic polymers and Cellulose with biopolymers. Under the synthetic polymers they have disussed the materials made with different versions of cellulose like Cellulose Nano Crystals, Cellulosic Whiskers, Cellulose Nano Fibres. Celluose with $\mathrm{SiO} 2$ and zeolite have also been discussed. Under biopolymers they have cited significant cellulose based bionanocomposites as good antibacterial agents for food packaging which eventually can be used in face masks. Ahmed et al (27) innovated reusable filtration system against SARS-CoV-2 consisting of a nanofibrous matrix of poly- lactic acid and cellulose acetate with copper oxide nanoparticles and graphene oxide nanosheets.

\section{Graphene based face masks}

Manoj Goswami et al (28) have reported the fabrication of the functionalized Graphene(fG) filter-based 3Dprinted face mask. These fG were prepared by Hummer's method. The fG filter shows $98.20 \%$ of bacterial filtration efficiency. The fG coated mask was tested against SARS-CoV-2 viral particles and showed excellent results. Flavio De Maio (29) worked with grapheme and graphene oxide integrated on cotton material and found it to possess potent antibacterial property. This suggested that these grapheme or grapheme oxide can be loaded on face mask or PPE, and even can be applied to trap infectious viral particles from effluents and municipalities, according to the authors. Stelbin Peter Figerez et al (30) designed and synthesized triple layer mask, containing nylon, modified polypropylene (PPY), and cotton nonwoven fabrics. The PPY was synthesized by melt- blown 
method and modified with graphene oxide and polyvinylidene fluoride mixture by solution casting method. The efficacy of this tri-layer system toward triboelectric rechargeability was discussed. These triboelectric nanogenerator (TENG)-assisted membranes were found to exhibit have high electrostatic charge retention capacity and high rechargeability in humid conditions. This material was found to have reusability as well. Hong Zhong et al (31) reported synthesis of mask coated with graphene fabricated by dual-mode laser scribing showed excellent hydrophobic and photothermal performance, reusability and self-sterilizing ability. They have shown antibacterial property as well. This mask contributes to the self-sterilization of viruses, which adhere to the surface for a long time, through solar illumination. The authors sound extremely positive by their view that after the virus is completely eradicated these masks can be recycled and reused for other purposes.

\section{Natural Products based Face masks}

Jatoi Abdul Wahab et al (32) fabricated SF/SESM (Silk Fibroin/Soluble Egg Shell Membrane) nanofibre composite, studied its water diffusion ability and water contact angle and found it to be very effective. Since this material is made from nature origin its disposal is quite eco-friendly. Bibaswan Sen et al (33) demonstrated that banana stem fibre extracted from banana peel can be used to make face mask. The advantage of this face mask is it can be recycled and biodegraded easily. According to this report transmission of bioaerosols is prevented to a larger extent when this face mask is impregnated with any microbe repelling material. Kajanan Selvaranjan et al (34) in their extensive review article have indicated exploitation of natural product based materials for face masks of polysaccharides, bamboo, hemp, coffee, sugar fibers, Tea leaf waste and sugarcane waste to make affordable and biodegradable green mask with maximum antibacterial efficiency and high capacity to filter. Currently many research studies are developing the required specification for biodegradable face masks.

\section{Electroststically charged face masks}

M. M. Bandi(35) synthesized electrochrged polypropylene(PP) mask as according to him when an electrostatically charged layer is sandwiched among standard mask filtration layers, oppositely charged particle are attracted by the long-range electrostatic Coulomb force and held by vander waals force. Interestingly the fibres are generated using modified cotton candy machine. He suggested the use of polymers obtained from recycled PET bottles or other polymers instead of PP.

\section{Next Generation Face masks}

Jialiang Zhou et al(36) in their review about antiviral protective material where they have mentioned about Organic antiviral materials which is further classified into photodynamic and intrinsic antiviral materials aprt from regular antiviral materials which is worth to be discussed. Sumit Kumar et al (37) synthesized Photoactive Antiviral Face Mask with Self-Sterilization in the presence of sunlight. Their nanocomposite included shellac and CuNP. The most important aspect of yhis material being its increase in temperature to $70 \mathrm{oC}$ under sunlight and free radical generated mechanism of self-cleaning.Si-Wei Xiong et al(38) reported the synthesis of Boron Nitride(BN) and polypropylene(PP) nanocomposite fibrous membrane for face mask which increses the wearing time, owing to its excellent heat dissipation from the surface of the mask eventually giving more comfort to the wearer. Since its antibacterial property is $99 \%$ it will serve as a good option for the face mask.Endre Horváth,et al (39)synthesizes TiO2NanoWires and demonstrated that when these nanowires were exposed to UV light generated surplus Reactive Oxygen Species(ROS) which resulted in its antimicrobial property. And hence can be used as a good photocatalytic filter. The authors suggest that this material can be produced in large scale and used as PPE material.

Luciano De Sio et al (40) have discussed about the synthesis of photothermal agents in face masks. They observed that organic dye molecules known asPhotosensitizers like indocyanine green, porphyrins, naphthalocyanines, are good choices for this role. It has been established that organic, metalbased-nanomaterials have proven theirpotential as photothermal generators due to their optical property. Peter Q. Nguyen et al (41) propose the use of Freeze Dried, Cell Free (FDCF) genetic circuits with specifically designed flexible materials and textile to make wearable bio sensors. They reported designs of various wearable FDCF sensors for small molecule, nucleic acid and toxin detection. The sensors are combined to form flexible multi-material substrates like textiles using genetically engineered components. Their main advantage being flexibility, elasticity and 
liquid absorbability through capillary action. The authors used FDCF sensors in wearable woven fabrics and individual threads for which they screened hundreds of fabrics of all types. This sensor-based mask could detect the pathogens to a good degree. With this study the authors have established that it is possible to make wearable genetically programmed sensor-based masks with good shelf stability and high sensitivity. They admit that the only drawback of this material being its non-recyclability and less effective in highly humid environment. Giulio Maria Bianco and Gaetano Marrocco (42) designed and tested N95 face mask fitted with Radio Frequency Identification Tag in a chip which can sense the difference between dampness in normal time and during physical excericise. This, the authors consider as an important parameter which could help further research in the making of PPE material. Vishal Chaudhary et al (43) have discussed the effects of graphene oxide, graphite and reduced grapheme oxide nanoparticles, Carbon nanodots, silica nanoparticles, nanoclay and its Multiwall carbon nanotubes as antiviral agents. Their study covers a wide range of topics like nanofibrous membrane based masks, self-refreshing $\mathrm{TiO} 2$ nanoparticles based and nanocomposite based masks. A. K. Aggarwal et al. have developed nanosilver-based coating for the fabrication of triple-layer medical mask.

\section{Reusability of face masks}

Cristina Crespo et al (44) colleagues developed new and simplified procedures for recycling FFP2 face masks used during Covid and assessed the chemical, thermal, and mechanical properties of the materials produced. The substance is a mix of polypropylene, polyethylene, and polyethylene terephthalate (PP, PE, and PET). The blend's mechanical qualities allow it to be used to produce a variety of goods, including flower pots, storage bins, shipping pallets, and toys. Sana Ullah et al (45) showed a comparative evaluation on the reusability of melt-blown and nano-fibre, using simple ethanol cleaning method. Subjecting the masks to repeated extensive characterizations like, airflow rate, filtration efficiency, morphological and surface properties, they reported that the nano-fibre masks had excellent reusability property.

\section{Conclusion}

Since face masks have proven to be the time-tested solution in preventing the airborne, droplet, and aerosol transmission of pathogenic microorganism during the COVID-19 pandemic, there is an overwhelming demand for antimicrobial face masks in the world market. To meet the demand for increasing requirement of face masks, several types of face masks have been designed and synthesized. The key factors to be considered by the researchers while designing the face mask apart from its antibacterial resistance are, wearability/comfort, breathability, reusability/multiple use, biodegradability, affordability and most importantly recyclability.

Therefore self-disinfecting and sensor based biodegradable masks made especially with natural plant or vegetable waste materials would be the best in the present scenario.

\section{References}

1) Wafa K Essa , Suhad A Yasin , Ibtisam A Saeed and Gomaa A M Ali , Membranes,11,250.

2) Mike Tebyetekerwa, Zhen Xu, Shengyuan Yang, Sreeram Ramakrishna, Advanced Fiber Materials, 2020.

3) Sabit Adanur and Ajay Jayswal, Journal of Industrial Textiles,2020,0,1-35.

4) Ilaria Armentano et al,Applied Polymer Materials,2021,3,531-548.

5) Gayathri Pullangott,Uthradevi Kannan, Gayathri S, Degala Venkata Kiran and Shihabudheen M Maliyekkal, RSC Advances,2021,11,6544.

6) Kris O’Dowd, Keerthi M. Nair, Parnia Forouzandeh, Snehamol Mathew , Jamie Grant, Ruth Moran, John Bartlett, Jerry Bird and Suresh C. Pillai, Materials, 2020.

7) Oluniyi O. Fadare and Elvis D. Okoffo, Science Of The Total Environment,2020.

8) Ming Hui Chua et al, Research,2020.

9) Padryk Merkl, Siwen Long, Gerald M. McInerney and Georgios A. Sotiriou , Nanomaterials, $2021,11,1312$

10) Melissa S Blevens, Homero F Pastrana, Hannah C Mazzotta, and Candace Su-Jung Tsai, Chemical Health and Safety,2021,28,171-182.

11) Abhishek Kumar et al, Advanced Functional Materials, 2021,31.

12) Sunghoon Jung et al, Polymers 2021, 13, 1367. 
13) Motahira Hashmi, SanaUllah, Ick Soo Kim, Current Research In Biology, 2019.

14) Chaitanya B. Hiragonda, Anuraj S. Kshirsagara, Vividha V. Dhapteb, Tanaya Khannaa, Prasad Joshia, Priyesh V. More, Vacuum, 2018.

15) Affaf Khamis Aloufy and Magdi Abdel-Moneim El-Messiry, Advances in Nano, Biomechanics,Robotics and Energy Research,2013,25-28

16) Benjamin Valdez-Salas, Ernesto Beltran-Partida,Nelson Cheng, Jorge Salvador-Carlos, Ernesto Alonso Valdez- Salas, Mario Curiel-Alvarez, Roberto Ibarra-Wiley, International Journal of Nanomedicine, 2021,16,2689-2702

17) Gownolla Malegowd Raghavendraa, Jeyoung Junga, Dowan kima, Jongchul Seoa, International Journal of Biological Macromolecules,2016.

18) Aniruddha Adhikari, ACS Applied Bio Materials, doi: 10.1021/acsabm.1c00238

19) Vigneshwaran Shanmugam et al, Journal of Applied Polymer Science,2021.

20) Vahid Babaahmadi, Hooman Amid, Mohammadreza Naeimirad, Seeram Ramakrishna, Science of the Total Environment,2021.

21) Pintu Pandit, Subhankar Maity, Kunal Singha, Annu, Muhammet Uzun, Mehdihasan Shekh, Shakeel Ahmed, Cleaner Engineering and Technology, 2021,(4),100218

22) Sejin Choi et al, Advanced Science,2021,8,2003155.

23) Morganti P and Morganti G, Journal of Health Care and Research, 2021, 1(3) 1(3):157-65.

24) C Akduman, Journal of Industrial Textiles,2019,(0),1-23.

25) Alyne R. de Araújo , Nanomanufacturing,2021,1,57-66

26) Yogesh Khairnar, Dharmesh Hansora , Chinmay Hazra , Debasree Kundu , Saurabh Tayde , Shyam Tonde Jitendra Naik, Aniruddha Chatterjee, Carbohydrate Polymer Technologies and Applications, 2021.

27) M.K. Ahmed,, A.A. Menazea,, S.F. Mansourd, Reem Al-Wafie,2020, Journal of Materials Research and Technology,2020, 9(5),11629-40.

28) Manoj Goswami et al, Journal of Science: Advanced Materials and Devices,2021.

29) Flavio De Maio et al,iScience,2020.

30) Stelbin Peter Figerez, Sudeshna Patra, G. Rajalakshmi and Tharangattu N. Narayanan, Oxford Open Materials Science, 2021, 1(1).

31) Hong Zhong, Zhaoran Zhu, Jing Lin, Chi Fai Cheung, Vivien L. Lu, Feng Yan, Ching-Yuen Chan,and Guijun Li, ACSNano, 2020.

32) Jatoi Abdul Wahab, Gang Xu1, Hoik Lee1, Phan Duy Nam1, Kai Wei, Seong Hun Kim3, and Ick Soo Kim, Fibers and Polymers 2016, 17(11), 1776-1781

33) Bibaswan Sen, Sayantani Paul, Kanchan Kumar Bhowmik, Surya Narayan Pradhan, Sk Imran Ali, Letters in Applied NanoBioScience, 2021, 10, 1993 - 2002

34) Kajanan Selvaranjan , Satheeskumar Navaratnam , , Pathmanathan Rajeev , Nishanthan Ravintherakumaran, Environmental Challenges,2021,3, 100039.

35) M. M. Bandi, Proc. R. Soc.,2020.

36) Jialiang Zhou, Zexu Hu, Fatemeh Zabihi, Zhigang Chen, Meifang Zhu, Advanced Fiber Materials,2020.

37) Sumit Kumar, Mamata Karmacharya, Shalik Ram Joshi, Oleksandra Gulenko, Juhee Park, Gun-Ho Kim, and Yoon-Kyoung Cho,NanoLetters, 2020.

38) Si-Wei Xiong et al, Applied Materials and Interfaces,2020.

39) Endre Horváth, Lídia Rossi, Cyprien Mercier, Caroline Lehmann, Andrzej Sienkiewicz, and László Forró, Advanced Functional Materials,2020.

40) Luciano De Sio et al, Chemistry europian Journal,2021, 27,6112-30.

41) Peter Q. Nguyen et al, Nature Biotechnology,2021.

42) Giulio Maria Bianco and Gaetano Marrocco,Sensors Letters, 2017,2(3)

43) Vishal Chaudhary, Abhishek Royal, Murthy Chavali and S. K. Yadav, Nanotechnology for Environmental Engineering,2021.

44) Cristina Crespo, Gemma Ibarz, Carlos Sáenz,Pablo Gonzalez,Sandra Roche,Waste and Biomass Valorization,2021.

45) Sana Ullah, et al , ACS Appled Nano Materials, 2020. 